\title{
Versuche über die Funktion der 1. Antenne von dekapoden Krebsen als Strömungssinnesorgan
}

\author{
Von W. Luther und H. Maier \\ Aus dem Zoologischen Institut der Technischen Hochschule Darmstadt
}

(Mit 2 Abbildungen in Text)

\section{Einleitung}

Die 1. oder "kleine“ Antenne der dekapoden Krebse ist in den Jahren 1926-39 mehrfach Gegenstand sinnesphysiologischer Untersuchungen gewesen (Brock 1926, Pagurus; Spiegel 1927, Crangon; Wrede 1928, Eupagurus; Luther 1930, Carcinus; van Heerdt und Krijgsman 1939, Eriocheir). Die eigentümlichen Sinneshaare auf der Außengeißel dieses Organs (sog. Leydigsche Sinneshaare) werden von älteren Autoren meist als Organe des chemischen Sinnes aufgefaßt („Riechhaare“). SpIEGEL (1927) und unabhängig davon Luther (1930) konnten jedoch übereinstimmend zeigen, daß sowohl bei dem Macruren Crangon crangon, wie bei dem Brachyuren Carcinus maenas bei isolierter Reizung der kleinen Antenne niemals eine Nahrungsreaktion eintritt. Bei höheren Reizstoffkonzentrationen beobachtet man bei Crangon eine allgemeine Unruhe (Putzbewegungen, Aufrichten aus dem Sand), während Carcinus bei Berührung der 1. Antennen mit einem Fleischstückchen diese sogar einzieht. Dagegen gelingt es leicht, bei Carcinus durch isolierte Reizung einer Fußspitze eine vollständige Beutefangreaktion auszulösen. Bei der Wollhandkrabbe, Eriocheir sinensis beobachteten van Heerdt und Krijgsman, daß Änderungen im pH-Wert des umgebenden Wassers (verwendet wurden $\mathrm{CO}_{2}$ und $\mathrm{HCl}$ ) eine kurzdauernde Stockung der Atembewegungen hervorrufen. Diese Reaktion blieb aus, wenn die 1. Antennen amputiert wurden. Die Verfasser nehmen daher an, daß der Krebs die Säure mit Hilfe von Chemorezeptoren wahrnimmt, die auf der 1. Antenne lokalisiert sind. Weitere Versuche mit der südafrikanischen Languste, Jasius lalandii (Krijgsman und Krijgsman 1954) ergaben lebhafte Flucht- und Abwehrreaktionen, wenn die kleine Antenne der Tiere mit osmotisch wirksamen Substanzen $(4 / 5-3 / 4$ verdünntes Seewasser) beträufelt wurden.

In neuester Zeit konnte Hodgson (1956, zit. nach Waterman 1961) bei Cambarus nach Reizung mit Zucker und Riechstoffen aus den Fußspitzen, den Mundwerkzeugen und auch aus der kleinen Antenne Aktionsströme ableiten. Die Ausschläge waren jedoch bei der kleinen Antenne deutlich schwächer als bei den übrigen Organen. 
Es kann somit als erwiesen angesehen werden, daß die Sinnesorgane der kleinen Antenne durch chemische Veränderungen des sie umgebenden Wassers erregt werden. $\mathrm{Ob}$ es sich dabei um eine echte Chemorezeption, d. h. um ein Erkennen und Unterscheiden bestimmter Stoffe handelt, könnte nur durch Dressurversuche bewiesen werden. Buddenbrock, der in seinem Buch „Vergleichende Physiologie" Bd. 1 (1952) die bis 1950 erschienenen Arbeiten zu dieser Frage ausführlich diskutiert hat, ist mit Spiegel (1928) der Meinung, die 1. Antenne sei ein "Alarmorgan“, das allein zwar nicht genügt, um gerichtete Suchhandlungen auszulösen, das aber in Zusammenarbeit mit anderen Chemorezeptoren die Reaktionsbereitschaft der Tiere erhöht.

Eine Alarmierung durch Addition verschiedener Sinnesreize setzt jedoch nicht voraus, daß diese Reize alle der gleichen Sinnesqualität angehören müssen. Auch Sinnesreize verschiedener Qualitäten (z. B. Geruch und Bewegungsweise des Beutetieres) können sich addieren und dadurch eine Fanghandlung auslösen. Nun ist von Luther (1930) gezeigt worden, daß die kleine Antenne von Carcinus in erster Linie als Strömungssinnesorgan funktioniert. Carcinus maenas geht, wie viele Tiere, bei der Futtersuche der Wasserströmung entgegen (positive Rheotaxis). Auf der Flucht läuft er, negativ rheotaktisch, mit dem Strom in tieferes Wasser. Amputiert man die 1. Antenne, so hört die rheotaktische Orientierung auf, die Krebse laufen ungerichtet stromauf, stromab oder quer zur Strömung. Das Vorhandensein bzw. Fehlen von Strömungswahrnehmungen könnte aber sehr wohl die Reaktionsbereitschaft der Tiere verändern und sie bei gleichzeitiger Reizung der übrigen Chemorezeptoren durch spezifische Riech- oder Schmeckstoffe zur Futtersuche veranlassen, auch wenn diese allein noch nicht genügen, den Krebs zum Verlassen seines Versteckplatzes zu veranlassen.

Über das rheotaktische Verhalten und den Sitz des Strömungssinnes bei anderen Dekapoden ist nur wenig bekannt. Es erschien uns daher lohnend, die nun schon über 30 Jahre zurückliegenden Versuche von Brock, SPIEgEL und Luther noch einmal wiederaufzunehmen, zunächst nur mit dem Ziel, die Bedeutung der kleinen Antenne als Strömungssinnesorgan für eine größere Zahl verschiedener Arten von Dekapoden nachzuprüfen. Da die Außengeißel der 1. Antenne bei Macruren, Anomuren und Brachyuren morphologisch recht verschieden gebaut sein kann, wurde je eine Art aus jeder der drei genannten Gruppen auf ihr Verhalten gegen Wasserströmungen geprüft.

\section{Methodisches}

Die Versuche wurden in einem Strömungskanal durchgeführt, in dem eine in sich geschlossene Wassermenge durch ein außerhalb des Versuchsraumes angebrachtes Schaufelrad in gleichmäßige Bewegung versetzt wurde. Die Konstruktion des zuletzt verwendeten Modells zeigt Abb. 1. Der ganze Apparat ist aus $5 \mathrm{~mm}$ starkem Plexiglas gebaut. Ein zu Versuchen mit Carcinus maenas und Cambarus affinis verwendetes älteres Modell unterschied sich im Prinzip nicht wesentlich von dem hier gezeigten, außer, daß dabei Metall als Baumaterial verwendet worden war.

Der $20 \times 60 \mathrm{~cm}$ große Versuchsraum ist durch 2 perforierte Zelluloidgitter abgeschlossen, die die Turbulenz des Wassers abfangen und für eine gleichmäßige langsame Strömung ohne "tote Winkel" sorgen. Die Rückwand besteht aus schwarzem Plexiglas, so daß die Tiere das Antriebsrad und die 
Transmissionen nicht sehen können. Zu den Versuchen wurde auch die Vorderseite des Apparates mit dunklem Papier verkleidet, um eine Beunruhigung der Tiere zu vermeiden. Der Apparat wurde so aufgestellt, daß das Licht möglichst gleichmäßig von oben oder quer zur Laufrichtung einfiel. Durch Umschalten des Motors konnte die Stromrichtung beliebig oft gewechselt werden. Die Strömungsgeschwindigkeit betrug etwa $3-3,5 \mathrm{~m} / \mathrm{min}$.

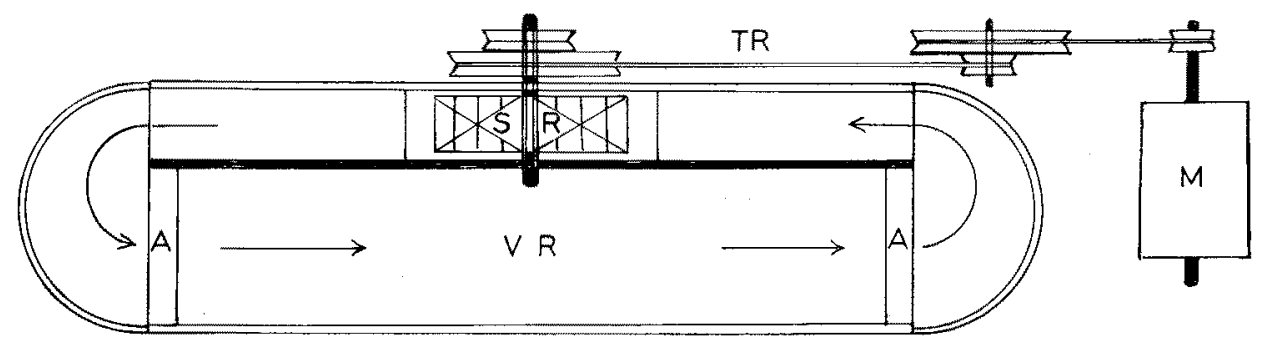

Abb. 1. Schematische Darstellung des für die Versuche mit Crangon und Clibanarius verwendeten Strömungsapparates. $\mathrm{A}=$ Absperrgitter, $\mathrm{M}=$ Motor (umschaltbar), $\mathrm{SR}=$ Schaufelrad zur Erzeugung des Wasserstromes, $\mathrm{TR}=$ Transmission

Wahl der Versuchsobjekte

Wie bei allen Arbeiten, bei denen das spontane Verhalten eines Versuchstieres als Kriterium benutzt werden soll, sind der Anwendbarkeit einer derartigen Methode natürliche Grenzen gesetzt durch die biologische Umwelt des zu untersuchenden Tieres und seine daraus resultierenden angeborenen Verhaltensweisen. Nur solche Tierarten, die sich auch normalerweise im Freien nach Wasserströmungen orientieren, sind für Rheotaxisversuche geeignet. Das beweist jedoch nicht, daß Tiere aus stehenden Gewässern oder größeren Meerestiefen Strömungsreize nicht wahrnehmen könnten. Man wird vielmehr, gleichen morphologischen Bau des Organs vorausgesetzt, annehmen dürfen, daß das, was für eine Art bewiesen werden konnte, auch für andere nahe verwandte Arten gilt. Es ist daher als ein glücklicher Umstand anzusehen, daß es, wie aus dem Folgenden noch hervorgehen wird, gelungen ist, die Bedeutung der kleinen Antennen für den Strömungssinn bei je einem Brachyuren, einem Anomuren und einem Macruren sicher nachzuweisen.

Die Versuche wurden teils in Darmstadt am Zoologischen Institut der Technischen Hochschule durchgeführt, teilweise auch an der Meeresbiologischen Station in Banyuls sur Mer (Frankreich) und der Sylter Wattenmeerstation der Biologischen Anstalt Helgoland. Den Leitern und dem Personal der beiden zuletzt genannten Stationen sind wir für ihre Unterstützung zu besonderem Dank verpflichtet.

\section{Beschreibung der Versuche \\ (vgl. hierzu die Tabelle auf S. 329)}

\section{Carcinus maenas $\mathrm{L}$.}

In den Jahren 1956-1957 wurden die schon fast 30 Jahre zurückliegenden Versuche von Luther (1930) an Carcinus maenas wiederholt (Staatsexamensarbeit H. MaIER, unveröffentlicht). Wie Luther schon damals beob- 
achten konnte, ist $C$. maenas bei der Futtersuche positiv rheotaktisch, bei der Flucht läuft er mit dem Strom. Die Versuche von MaIer mit der neuen Apparatur bestätigen diese Beobachtung. Wenn dem Wasser im Strömungskanal vor Beginn der Versuche eine geringe Menge Fleischsaft zugesetzt worden war, liefen sämtliche 10 untersuchten Tiere in mehr als 300 Versuchen aufgeregt suchend der Strömung entgegen. Wurde die Stromrichtung umgekehrt, so änderte auch die Krabbe sofort ihre Laufrichtung. Der Wechsel der Stromrichtung konnte in den meisten Fällen $4-8 \mathrm{mal}$ wiederholt werden. Nur ganz vereinzelt kam eine negative Reaktion vor $(2 \%)$ oder das Tier lief überhaupt nicht, sondern grub sich ein $(1 \%)$. Solche Tiere wurden für den Rest des Versuchstages ausgeschieden. Am darauffolgenden Tage reagierten auch sie wieder positiv.

Versuche in reinem Wasser ohne Zusatz von Futtersaft zeigten, daß hungrige Carcinus auch ohne chemische Alarmierung positiv rheotaktisch sind. $82 \%$ der Läufe waren positiv, $17 \%$ negativ und $1 \%$ unsicher. Hier genügt offenbar schon das Umsetzen allein, um die ewig hungrigen Krabben zum Absuchen der neuen Umgebung zu veranlassen.

Amputiert man die 1. Antenne, so ändert sich das Verhalten in ganz charakteristischer Weise. Die amputierten Krebse suchen zwar ebenso eifrig nach Futter wie unoperierte Tiere, sie laufen dabei aber völlig ungerichtet hin und her, wechseln immer wieder spontan die Richtung oder graben längere Zeit an ein und derselben Stelle im Sand. Die zahlenmäßige Auswertung von 100 Versuchen ergab 58 mal Laufrichtung gegen den Strom, 42 mal liefen die Krabben mit der Strömung. Anderungen der Stromrichtung wurden kaum beachtet. Nur in 5\% der Fälle kam es vor, daß die Krabbe beim Umschalten der Stromrichtung ebenfalls umkehrte. Dabei ist nicht ausgeschlossen, daß es sich um Fälle von spontanem Richtungswechsel handelt.

Strandkrabben, denen nur eine der beiden kleinen Antennen amputiert worden war, verhielten sich rheotaktisch genauso wie unoperierte Tiere.

Im Gegensatz zu den futtersuchenden Tieren flüchten aufgeschreckte, satte Carcinus fast immer stromab. Es ließ sich zeigen, daß auch diese negative Rheotaxis von der kleinen Antenne aus gesteuert wird. Zu diesem Zweck wurden in der Mitte des schon beschriebenen Strömungskanals zwei Elektroden eingebaut, zwischen denen beim Niederdrücken einer Taste ein kurzer Stromstoß (4 V, 0,24 A) ausgelöst werden konnte. Die Krabben erwiesen sich als sehr empfindlich gegen elektrischen Strom. Ein zwischen den Elektroden hindurchlaufendes Tier wurde durch plötzliches Einschalten sofort zu heftigster Flucht veranlaßt.

Die Versuche wurden mit frischgefangenen Krabben durchgeführt, die außerdem reichlich gefüttert worden waren. Die positive Rheotaxis ist bei diesen Tieren weniger stark ausgeprägt, als in den vorhergehenden Versuchsreihen. Nur etwa $74 \%$ liefen beim Einsetzen spontan gegen den Strom, 26\% gingen spontan stromab. Wurde der Strom für etwa $1 / 4 \mathrm{sec}$. eingeschaltet, so fïüchteten $75 \%$ der Tiere stromab und nur $25 \%$ liefen in der ursprünglichen Richtung stromauf weiter.

Nach der Amputation der kleinen Antennen war ein Einfluß der Wasserströmung auf die Fluchtrichtung nicht mehr nachzuweisen. Die gegen den Strom marschierenden Tiere behielten auch nach dem Schock die einmal eingeschlagene Richtung bei. Die Auszählung ergab 52\% Flucht stromauf und $48 \%$ Flucht stromab. 
Auch bei der Fluchtreaktion wird somit die Bewegungsrichtung der Tiere von der kleinen Antenne aus gesteuert.

\section{Clibanarius misanthropus Risso}

Der Felsküsten-Einsiedler, Clibanarius misanthropus Risso, lebt im Mittelmeer auf Blockgründen der obersten Flachwasserzone in $0,5-2 \mathrm{~m}$ Tiefe. In diesem Gebiet laufen die Wellen über den Steinen an der Oberfläche auf die Küste zu, das zurückflutende Wasser strömt unter und zwischen den Blökken ins Meer zurück. Bei Störungen flüchten die Einsiedlerkrebse mit der Unterströmung tiefer gelegenen Schlupfwinkeln zu. Diese Reaktion ermöglicht eine Untersuchung des Strömungssinnes, ohne daß dazu die bei Anomuren sehr viel weniger deutlichen Futterreaktionen herangezogen werden müssen.

Bringt man eine größere Anzahl von Felsküsteneinsiedlern (10-15 Stück) in die Mitte des Durchströmungsraumes unserer Versuchsapparatur, so kommen sie schon nach einigen Sekunden aus ihren Häuschen hervor und beginnen nach allen Richtungen auseinanderzulaufen. Sehr bald jedoch beobachtet man, daß die stromaufgehenden Tiere anhalten, durch lebhaftes Schlagen mit den kleinen Antennen die Stromrichtung prüfen und dann umkehren und mit dem Strom gehen. Nur ein verhältnismäßig kleiner Teil behält die einmal eingeschlagene Richtung stromaufwärts bei bis zum oberen Absperrgitter. Am oberen wie am unteren Gitter verweilen die Krebse meist längere Zeit. Sie untersuchen die Perforationslöcher mit Füßen und Scheren oder klettern am Gitter empor. Nach einigen Minuten sind alle in Bewegung befindlichen Tiere an den Enden des Versuchskanals versammelt.

Die Auszählung jeweils 5 min. nach Versuchsbeginn ergab eine sehr starke Bevorzugung des stromab gelegenen Gitters. In 35 Versuchsserien, die an verschiedenen Tagen mit über 50 Einzeltieren durchgeführt wurden, sammelten sich 304 Krebse am unteren Gitter und nur 110 am oberen. 58 Tiere wurden nicht registriert, weil sie entweder überhaupt nicht liefen oder sich noch vor Erreichung des Gitters im Sand zur Ruhe setzten. Läßt man diese außer acht, so ergibt sich ein Zahlenverhältnis von $73,7 \% \mathrm{zu} 26,3 \%$ für die Richtung stromab.

Amputiert man nun beide kleinen Antennen, so hört ebenso wie bei Carcinus die rheotaktische Orientierung auf. Gezählt wurden 456 Läufe von 25 amputierten Tieren. Das Ergebnis war: 160 Tiere am oberen und 161 am unteren Gitter, also fast genau 50:50\%. Dabei war sehr deutlich zu beobachten, daß die antennulenlosen Einsiedlerkrebse ebenso wie amputierte Carcinus immer wieder die Richtung wechseln und scheinbar planlos an ein und derselben Stelle umhersuchen. 135 Tiere, d.h. fast ein Drittel der Gesamtzahl, konnte überhaupt nicht gewertet werden, weil sie in der Mitte des Beckens blieben und das Gitter nicht erreichten. Da die operierten Tiere sich sonst ganz normal verhielten und gut fraßen, kommt ein Wundschock als Ursache für das veränderte Verhalten im Wasserstrom kaum in Frage. Wir müssen vielmehr annehmen, daß sie, ebenso wie die Brachyuren, die Wasserströmung nach Amputation der Antennen nicht mehr wahrnehmen und sich dementsprechend verhalten wie in einem Becken mit stehendem Wasser. 


\section{Eupagurus bernardus L.}

Von Jungtieren des Nordsee-Einsiedlers Eupagurus bernardus L. sollte man erwarten, daß sie sich als Bewohner des Wattenmeeres ähnlich verhalten würden wie Clibanarius misanthropus. Versuche mit Tieren aus dem Flachwasser des Königshafens von Sylt ergaben jedoch ein negatives oder doch recht unsicheres Ergebnis. In 140 gezählten Läufen (35 Tiere) wurde $77 \mathrm{mal}$ das untere und $63 \mathrm{mal}$ das obere Gitter aufgesucht $(55 \%: 45 \%)$.

Versuche, die Fluchttendenz der Einsiedlerkrebse durch Wegnehmen der Schneckenhäuschen zu steigern, verbesserten das Ergebnis nicht. Auch geblendete Tiere reagierten nicht anders als normale. Daß auch antennulenlose Eupagurus keinerlei Strömungsorientierung erkennen ließen, ist unter diesen Umständen nicht verwunderlich. Die genauere Beobachtung des Freilandverhaltens zeigte dann auch, daß die Krebse zur Ebbezeit bei ablaufendem Wasser nicht abwandern, sondern sich halb vergraben im Sande festsetzen. Sie gehen auch nie weiter den Strand hinauf als bis zu einer Höhe, die auch bei Niedrigwasser nicht ganz trocken fällt. Erwachsene Eupagurus bernardus leben in tieferem Wasser bei $10-20 \mathrm{~m}$, wo die Gezeitenströme wahrscheinlich sowieso nur von geringer biologischer Bedeutung für sie sind. Damit ist jedoch keineswegs gesagt, daß sie Strömungen nicht wahrzunehmen vermögen. Es ist sehr wahrscheinlich, daß beim Auffinden der Nahrung der Strömungssinn beteiligt ist und sich unter entsprechenden Versuchsbedingungen auch im Experiment nachweisen läßt.

\section{Crangon crangon $\mathrm{L}$.}

Unter den macruren Decapoden erwies sich die Sandgarnele Crangon crangon L. als besonders geeignet für Strömungsversuche, weil diese Tiere schon in ihrem normalen Verhalten positive Rheotaxis zeigen. Sie wandern im Freien, sofern sie nicht eingegraben im Sand ruhen, mit jeder steigenden Flut langsam landwärts bis in die kleinsten Priele und Wattenmeertümpel hinein, in denen sie bei Ebbe nicht selten zurückbleiben. Jungtiere sind hierbei beweglicher und gehen weiter zum Strand hinauf als ausgewachsene Exemplare. $\mathrm{Zu}$ den im folgenden beschriebenen Versuchen wurden deshalb nur halberwachsene Exemplare von 3-4 cm Gesamtlänge verwendet.

Im Strömungskanal, wo ihnen die Möglichkeit sich einzugraben genommen ist, sind die Garnelen sehr unruhig. Einige suchen sofort das obere Absperrgitter auf und versuchen, wie Fische auf und ab schwimmend, dem einströmenden Wasser entgegen zu entkommen. Andere liegen mit dem Kopf der Strömung zugewendet am Boden und rücken in kurzen Sprüngen von 1-2 Körperlängen langsam stromauf. Vereinzelt beobachtet man auch solche, die mit dem Kopf stromab in der Strömung liegen oder Tiere, die mehr oder weniger zufällig ans untere Gitter gelangt sind und versuchen dort durchzukommen. Dagegen sieht man nur sehr selten eine normale Sandgarnele schräg zur Stromrichtung liegen. Wird ein solches Tier mit einem Grashalm aufgescheucht, so wendet es sich fast immer sofort dem einströmenden Wasser zu.

Zur Auswertung der Versuchsergebnisse wurden die am oberen Gitter versammelten und die mit dem Kopf stromauf liegenden Tiere 5 min. nach Einschalten der Strömung registriert und dieser Wert der Zahl der stromab gerichteten oder am unteren Gitter versammelten gegenübergestellt. Außer- 
dem wurde die Verteilung aller Tiere zeichnerisch in einem Schema festgehalten. Dann wurde der Strom umgekehrt und die Garnelen durch Aufstören mit einem Strohhalm durcheinandergejagt. Nach etwa 4-5 Versuchen wurden sie herausgefangen und durch neue Tiere ersetzt.

Abb. 2 a zeigt einige typische Verteilungsbilder von unoperierten Crangon crangon. Die Zahlenauswertung aller Versuche ergab 220mal Ausrichtung gegen den Strom und $81 \mathrm{mal}$ mit dem Strom $(69 \%: 26 \%$ ). Schräglage kam nur $17 \mathrm{mal}$ vor (d. h. 5,3\% der Gesamtzahl).

Die Außengeißel der kleinen Antenne ist bei ruhenden oder schwimmenden Crangon senkrecht zur Körperachse aufgerichtet. Die Amputation der Antennulen kann bei einem im Sande vergrabenen Tier vorgenommen werden, ohne daß es dabei irgend ein Zeichen von Schmerz oder Unbehagen verrät. Das Verhalten im Strömungskanal ist jedoch bei den operierten Garnelen grundlegend verändert. Die Ansammlungen am oberen Absperrgitter bleiben aus; die Tiere sind weniger schwimmlustig als normale und liegen oft längere Zeit ruhig an einer Stelle. Dabei können sie jede beliebige Lage zur Strömungsrichtung einnehmen: Von 250 amputierten Tieren lagen 138, d. h. mehr als die Hälfte, quer oder schräg zum Strom, die übrigen 112 lagen fast genau gleich oft stromauf wie stromab (57:55). Abb. 2 b zeigt einige Beispiele dieser völlig regellosen Verteilung.

Daraus kann geschlossen werden, daß antennulenlose Crangon nicht mehr imstande sind, die Richtung eines Wasserstromes wahrzunehmen. Wenn die amputierten Tiere immer noch verhältnismäßig oft parallel zur Strömung
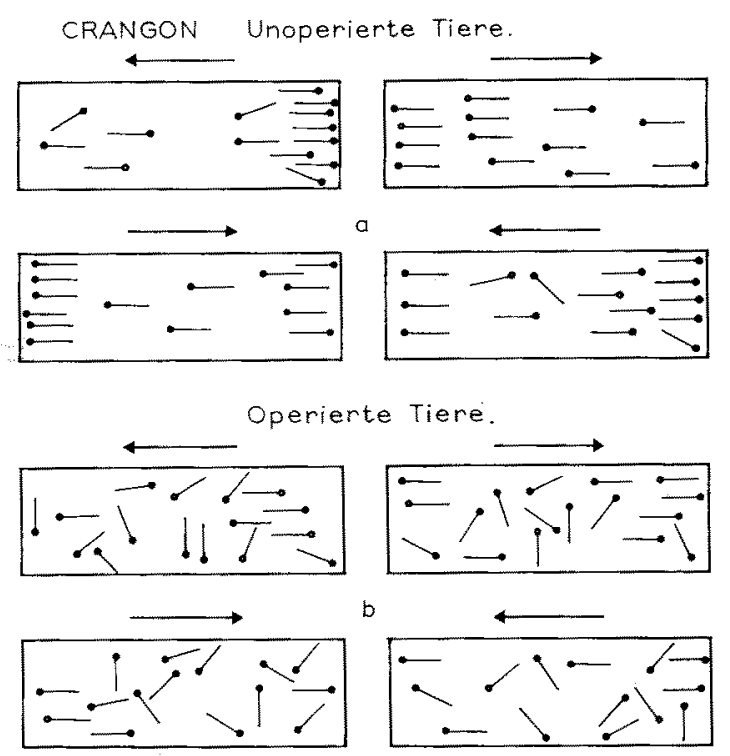

Abb. 2. Einzelbeispiele der Verteilung unoperierter (a) und antennulenamputierter (b) Sandgarnelen (Crangon) im Strömungskanal. $\rightarrow=$ Stromridhtung. Man beachte in a die gerade Ausrichtung auch der mit dem Kopf stromab liegenden Tiere

lagen $(45 \%)$, so kann dies durch die spindelförmige Gestalt des Krebses bedingt sein, der sich rein passiv in die Stromrichtung einstellt. Berührt man eine antennulenlose Garnele vorsichtig mit einem Grashalm, so rückt sie zur Seite, wobei sie sich meistens quer stellt, wäbrend ein unoperiertes Tier ent- 
weder fortschwimmt oder sich einige Zentimeter weiter wieder parallel ausrichtet. Die Spindelgestalt des Körpers wirkt also vielleicht begünstigend aber keinesfalls entscheidend auf die Richtungsorientierung des ruhenden Tieres.

\section{Palaemon serratus Pennant}

Versuche mit den Schwimmgarnelen des Mittelmeeres Palaemon serratus und P.xiphias verliefen völlig negativ. Die Garnelen schwammen quer zur Stromrichtung an der Vorderscheibe des Versuchsbeckens auf und ab und zeigten kein besonderes Interesse für das obere oder untere Absperrgitter. Biologisch ist das verständlich, denn im Lebensraum dieser Garnelen (Felsspalten, Höhlen und ruhige Buchten) sind die Wasserströmungen sehr wechselnd und unregelmäßig. Die Orientierung erfolgt hier wohl vorwiegend optisch oder taktil durch die sehr langen Fühleräste der 1. und 2. Antenne. Man kann auch im Freien beobachten, daß Schwimmgarnelen, die einen ausgelegten Köder suchen, sich in sehr weiten Zickzacklinien ihrem Ziele nähern (phobische Reaktion, Orientierung durch Versuch und Irrtum) während Strandkrabben meist ziemlich gradlinig darauf zulaufen.

\section{Cambarus affinis Say.}

Die am Boden lebenden langschwänzigen Dekapoden (Marcrura reptantia) zerfallen in 2 nach dem Bau ihrer 1 . Antennen deutlich getrennte systematische Gruppen. Bei den Langusten und Bärenkrebsen (Palinuridae, Scyllaridae) ist die 1. Antenne sehr ähnlich gebaut wie bei Anomuren und Brachyuren, mit langem Basalteil und sehr kurzer, mit einem dichten Kamm von Sinneshaaren besetzter Außengeißel. Untersuchungen über ihre Funktion liegen bisher nicht vor. Die Art, wie z. B. die Mittelmeerlanguste (Palinurus vulgaris Latr.), ihre 1. Antennen beim Futtersuchen über dem Beutestück hin und her bewegt, legt die Vermutung nahe, daß dieses Organ hier vielleicht neben seiner Funktion als Tastorgan auch noch Träger von chemischen Sinnesorganen ist. Bei der Größe der Tiere wäre eine nervenphysiologische Prüfung durch Ableiten von Aktionsströmen aus der isolierten Antenne wohl möglich.

Die 1. Antenne der Flußkrebse (Astacidae) ist ziemlich lang und peitschenförmig, die Leidigschen Sinneshaare sind kurz und wenig auffällig. Bei Süßwasserbewohnern war von vornherein zu erwarten, daß die Tiere sich in ihrem Lebensraum nach Wasserströmungen richten. Die Durchführung der Versuche (H. MAIER, Staatsexamensarbeit 1957, vgl. S. 323) erwies sich als nicht ganz einfach, weil Flußkrebse sehr viel anfälliger gegen äußere Störfaktoren sind als die viel robusteren Krabben und Garnelen. Der gewöhnliche Flußkrebs Astacus (= Potamobius) fluviatilis L. wurde daher nach einigen Versuchen ausgeschieden. Dagegen arbeitete der amerikanische Flußkrebs Cambarus affinis Say bei Beachtung einiger Vorsichtsmaßnahmen (längere Eingewöhnung der Tiere, knappe Fütterung, Wassertemperaturen unter $16^{\circ} \mathrm{C}$ ) im Strömungsapparat fast ebenso gut wie Carcinus maenas. Die Krebse zeigten bereits spontan eine individuell recht verschiedene aber deutliche Tendenz dem Strom entgegen zu laufen. Bei Zusatz von Fleischsaft zum Wasser wird diese positive Rheotaxis verstärkt: In 150 Versuchen, bei denen die Krebse mit dem Schwanzende zur Strömung in das Becken eingesetzt wurden, drehten sie sich 
$128 \mathrm{mal}$ sofort um und gingen stromauf $(85,5 \%)$. Umschalten der Stromrichtung wurde in 91 Fällen $(=60,8 \%)$ mit einer entsprechenden Kehrtwendung des Tieres beantwortet. Wurden die Krebse gleich in der "richtigen“ Richtung eingesetzt (mit dem Kopf stromauf), so stieg die Zahl der positiven Reaktionen sogar auf $97,5 \%$ von weiteren 150 Versuchen. Nur 4 mal wendete der Krebs sich stromab (2,5\%). Eine Kehrtwendung auf Umschalten des Stromes wurde bei den stromab gehenden Tieren nicht beobachtet. Cambarus affinis ist also ebenso wie Crangon stark rheotaktisch. Eigenartigerweise scheint jedoch hier das rheotaktische Verhalten nicht an das Vorhandensein der kleinen Antennen gebunden. Sowohl nach Amputation des Außenastes wie nach Entfernen der ganzen Antennula bis zum 2. Basalglied zeigten die Krebse noch deutlich gerichtetes Verhalten zur Strömung, mit den typischen Kehrtwendungen beim Umschalten der Stromrichtung. Eine zahlenmäßige Auswertung der Versuche mit amputierten Cambarus konnte nicht vorgenommen werden, da die Tiere kurz nach der Operation eingingen. Die wenigen protokollierten Versuche waren jedoch absolut eindeutig (MAien 1957). Cambarus affinis als Vertreter der sog. Macrura reptantia unterscheidet sich somit nicht nur von den Brachyuren und Anomuren, sondern auch von den sehr viel näher verwandten Garnelen (Macrura natantia) der Gattung Crangon, bei denen, wie wir sahen, die Orientierung zur Wasserströmung mit dem Verlust der 1. Antennen ganz oder doch fast ganz aufhört. Durch Heranziehung weiterer Arten (so vor allem des Hummers und der mit Crangon näher verwandten Alpheus-Arten) wird es vielleicht möglich sein zu prüfen, ob es sich hierbei um einen systematischen Gruppenunterschied handelt oder nur um eine spezielle Anpassung an das Leben im schnellfließenden Süßwasser.

Tabellarische Übersicht aller Strömungsversuche

\begin{tabular}{llllc}
\hline Tierart 1 Antenne & Reaktion & $\begin{array}{c}\text { Zahl } \\
\text { der Läufe }\end{array}$ & $\begin{array}{c}\text { Zahl } \\
\text { der Tiere }\end{array}$ & $\begin{array}{c}\text { Laufrichtung } \% \\
\text { stromauf stromab indiff. }\end{array}$ \\
\hline
\end{tabular}

Tab. I Brachyuren

\begin{tabular}{|c|c|c|c|c|c|c|c|}
\hline $\begin{array}{l}\text { Carcinus } \\
\text { maenas }\end{array}$ & normal & spontan & 100 & 10 & 82 & 17 & 1 \\
\hline d to. & normal & Futtersuche & 100 & 10 & 97 & 2 & 1 \\
\hline dto. & normal & Flucht & 100 & 10 & 25 & 75 & - \\
\hline $\begin{array}{l}\text { Carcinus } \\
\text { maenas }\end{array}$ & amputiert & Futtersuche & 105 & 9 & 58 & 42 & - \\
\hline dto. & amputiert & Flucht & 100 & 9 & 52 & 48 & 一 \\
\hline
\end{tabular}

Tab. II Anomuren

\begin{tabular}{|c|c|c|c|c|c|c|}
\hline $\begin{array}{l}\text { Clibanarius } \\
\text { misanthropus }\end{array}$ & normal & Flucht & 414 & 50 & 26,3 & 73,7 \\
\hline dto. & amputiert & Flucht & 456 & 25 & 49,8 & 50,2 \\
\hline $\begin{array}{l}\text { Enpagurus } \\
\text { bernardus }\end{array}$ & normal & Flucht & 140 & 35 & 45 & 55 \\
\hline
\end{tabular}




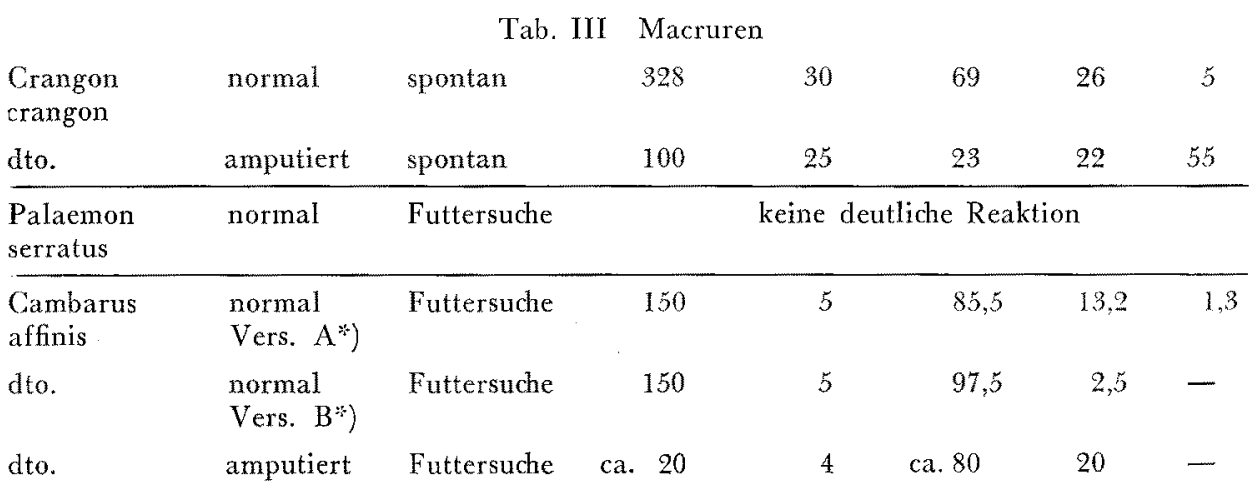

$\approx$ Vers. $A=$ Beim Einsetzen Kopf stromab. Vers. B = Kopf stromauf.

\section{Diskussion der Ergebnisse}

Auf S. 329/330 sind die Ergebnisse unserer Versuche in tabellarischer Form zusammengestellt. Zusammen mit den Beobachtungen von Krijgsman, Hodgson u. a. ergibt sich daraus, daß die kleine Antenne der Dekapoden ein recht vielseitiges Organ darstellt, das mechanische, osmotische und wenigstens bis zu einem gewissen Grade wohl auch chemische Reize aufnimmt und sie als Informationen an das Zentralnervensystem weiterleitet, wo sie mit den Informationen der übrigen Sinnesorgane integriert und zu einer biologisch sinnvollen Verhaltensweise verwendet werden. Die nächste Frage wäre nun, ob diese Informationen von ein und demselben Sinnesorgan, nämlich den Leydigschen Sinneshaaren, empfangen werden, oder ob, wie so häufig bei Arthropoden, verschiedene Arten von Sinneszellen und Sinnesapparaten nebeneinander vorkommen? Eine genaue vergleichend anatomische Untersuchung der Antennulen verschiedener Dekapoden steht noch aus. Die oberflächliche Betrachtung von Totalpräparaten in Glycerin zeigt, daß die Außengeißel der 1. Antenne von Crangon in jedem ihrer Glieder außer den Leydigschen Sinneshaaren nur noch rechts und links je ein Grüppchen sehr kurzer Tastborsten trägt. Die Innengeißel ist bei Crangon dicht mit langen Fiederborsten besetzt, die sicherlich Tastreize übertragen könnten. Diese Fiederborsten fehlen jedoch auf der sehr kurzen starren Innengeißel der 1 . Antenne von Carcinus. Da aber, wie wir gesehen haben, die Rheotaxis der Brachyuren und Anomuren ebenso wie bei Crangon an das Vorhandensein der 1. Antenne gebunden ist, können wir die Fiederborsten des Innenastes nicht als das entscheidende Organ des Strömungssinnes ansehen. Porenplatten, wie sie z. B, auf den Fußspitzen von Carcinus und auf den Riechfühlern der Insekten in großer Menge vorkommen, fehlen auf der kleinen Antenne der von uns untersuchten Dekapoden anscheinend ganz.

Ihrer Struktur nach wären die langen, sehr dünnwandigen Leydigschen Sinneshaare als Rezeptoren für chemische Reize durchaus geeignet. Sie sind deshalb auch von vielen früheren Untersuchern als "Riechhaare" aufgefaßt worden. Ebenso gut könnten sie natürlich auch als Organ eines osmotischen Sinnes funktionieren, wie er von KrIJgsman nachgewiesen worden ist. Es bleibt aber dann die Frage offen, wie und mit Hilfe welcher Organe die kleine Antenne der Brachyuren die Strömungsreize wahrnimmt. Daß sie wahrgenom- 
men werden, ist sicher - andererseits jedoch besitzt die kleine Antenne z. B. von Carcinus außer den Leydigschen Haaren keine irgendwie als Tastorgan spezialisierte Borsten, die als Rheozeptoren in Betracht kommen könnten.

Wir stehen somit vor der Frage, ob ein und dasselbe Sinnesorgan verschiedene Reizqualitäten gleichzeitig wahrnehmen und, was noch wichtiger ist, differenzieren kann. Eine derartige Annahme erscheint nach allem, was wir bisher über die Funktion von Sinneszellen wissen, im höchsten Grade unwahrscheinlich. Nun geht aber aus den Versuchen von Spiegel, KrJjgsman u. a. auch nicht sicher hervor, daß die von den 1 . Antennen wahrgenommenen chemischen und osmotischen Reize spezifisch sind. Man denke etwa an die hohe Empfindlichkeit der menschlichen Augenschleimhaut gegen ätzende Stoffe aller Art! Die soeben genannten Verfasser erhielten bei ihren Tieren außer einer allgemeinen Erregung (SpiEgel) nur Abwehrreaktionen (Putzen, Anhalten der Atmung oder sogar heftige Fluchtversuche). Auch die von Hodgson (vgl. WATERMan 1961, Seite 114/115) verwendeten 0,25 molaren Lösungen von Glycerin oder Glutaminsäure sind so hoch konzentriert, daß sie sehr wahrscheinlich neben einer chemischen Wirksamkeit auch osmotisch auf die Leydigschen Sinneshaare einwirken können. Eine Entscheidung darüber könnte wohl nur ein Versuch nach der von SPIEgel (1927) bei Crangon verwendeten Methode isolierter Reizung erbringen, wenn dieser mit Tieren durchgeführt würde, die vorher auf eine biologisch sonst unwirksame Substanz dressiert wurden. Wenn ein solcher, zunächst indifferenter Stoff nach der Dressur eine Erregung des Tieres bei Reizung der isolierten Antennen hervorruft, würde dies beweisen, daß der Stoff erkannt wird, d. h. daß eine echte Chemorezeption vorliegt.

Eine andere Frage ist noch, ob und wieweit die Spezialisierung bestimmter Organe des Tastsinnes zu einem einzigen rheorezeptorischen Organ eben der 1. Antenne - eine allgemein verbreitete Eigenschaft der dekapoden Krebse darstellt, oder nur eine Spezialität bestimmter, an das Leben in strömendem Wasser besonders angepaßter Arten. Da die rheorezeptorische Funktion der 1. Antenne bei Brachyuren, Anomuren und Macruren trotz ihres verschiedenen Baues in gleicher Weise auftritt, ist die erstere Annahme wahrscheinlicher. Wenn viele Arten in der von uns verwendeten Versuchsanordnung keine oder keine deutlichen Orientierungsbewegungen zur Wasserströmung zeigten, so bedeutet das keineswegs, daß sie die Strömung nicht wahrnehmen. Es ist sogar sehr wahrscheinlich, daß viele Arten, für die Wasserbewegungen normalerweise ohne Bedeutung sind, unter besonderen Bedingungen, z. B. bei der Futtersuche oder bei periodischen Wanderungen, positiv rheotaktisch reagieren würden. Es muß einer verbesserten, feineren Untersuchungsmethode überlassen bleiben, solche Bedingungen zu schaffen, um die Bedeutung der kleinen Antenne für diese gerichtete Reaktion zu prüfen.

\section{Zusammenfassung}

Verschiedene Decapoden (Carcinus maenas, Clibanarius misanthropus, Crangon crangon und Cambarus affinis) wurden auf ihr Verhalten gegen Wasserströmungen geprüft.

Carcinus, Crangon und Cambarus erwiesen sich dabei als spontan theotaktisch. Im Strömungskanal wandern sie dem Wasserstrom entgegen oder 
richten sich mit dem Kopf gegen die Strömung. Durch Zusatz von Futtersaft zum strömenden Wasser wird die Rheotaxis bei Carcinus und bei Cambarus deutlich verstärkt. Die Tiere suchen gegen den Strom laufend nach dem Futter.

Clibanarius misanthropus und Carcinus maenas verhalten sich bei der Flucht negativ rheotaktisch. Sie fliehen mit dem Strom und sammeln sich an der Ausflußöffnung des Strömungskanals an.

Bei Carcinus, Clibanarius und Crangon hört das rheotaktische Verhalten sofort auf, wenn die 1. Antenne amputiert wird. Antennulenlose Tiere zeigen keinerlei Orientierung nach der Stromrichtung mehr. Es wird daraus geschlossen, daß die kleine Antenne der Dekapoden ein Organ des Strömungssinnes ist.

Eine Ausnahme macht der Flußkrebs Cambarus affinis Say. Hier bleibt die rheotaktische Einstellung auch nach Amputation beider Antennulen erhalten. Die Organe des Strömungssinnes sind also bei Cambarus nicht auf die kleine Antenne allein beschränkt.

Eupagurus bernardus und die Schwimmgarnelen Palaemon serratus und $P$. xiphias zeigten keine Orientierungsreaktionen zur Stromrichtung. Das kann auf eine für diese Arten ungeeignete Versuchsanordnung zurückzuführen sein, oder darauf, daß im natürlichen Lebensraum dieser Tiere Wasserströmungen keine oder nur geringe biologische Bedeutung haben.

\section{Schriftenverzeichnis}

Brock, F.: Das Verhalten des Einsiedlerkrebses Pagurus arrosor Herbst, während der Suche und Aufnahme der Nahrung. Z. Morph. u. Okol. d. Tiere 6 (1926).

B rock, F.: Das Verhalten der ersten Antennen von Brachyuren und Anomuren in bezug auf das umgebende Medium. Z. vergl. Physiol. 11 (1930).

B rock, F.: Kritische Bemerkungen zu einer Arbeit Wolfgang Luthers, Versuche über die Chemorezeption der Brachyuren. Zool. Anz. 92 (1950).

Buddenbrock, W. von: Vergleichende Physiologie Bd. I: Sinnesphysiologie. Basel 1952

Buddenbrock, W. von: Physiologie der Dekapoden in Bronns "Tierreid" Bd. 5 Abt. 1, Leipzig 1945.

Hodg son, E. S.: Electrophysiological studies on arthropod chemoreception III. Chemoreceptors $\mathrm{f}$ terrestrial and fresh water arthropods. Biol. Bull. 115, 1958 (zit. nad Water$\operatorname{man} 1961$ ).

Krijgsman, B. I. und Krijgsman, N. E.: Osmoreception in Jasius lalandii. Z. vergl. Physiolog. 37 (1954).

Luther, W.: Versuche über die Chemorezeption der Brachyuren. Z. vergl. Physiol. 12 (1930).

Luther, W.: Zur Frage der Chemorezeption der Brachyuren und Anomuren. Zool. Anz. 94 (1931).

M a i e r, H.: Untersubungen über den Strömungssinn bei höheren Krebsen. Staatsexamensarbeit, Zool. Institut der TH Darmstadt, 1957 (unveröff.).

Prosser, C. L. und Brown, F. A.: Comparative animal physiology. II. Aufl. Philadelphia und London, 1962.

S p i e g e 1, A.: Über die Chemorezeption von Crangon vulgaris. Z. vergl. Physiol. 6 (1927)

Van Heerdt, P. F. und Krijgsman, B. J.: Die Regulierung der Atmung bei Eriocheir sinensis. Z. vergl. Physiol. 27 (1939.)

W aterman, T. H.; The physiology of Crustacea, Bd. II. New York und London, 1961 